\title{
OCTONION PLANES IN CHARACTERISTIC TWO
}

\author{
BY JOHN R. FAULKNER ${ }^{1}$
}

Communicated by Nathan Jacobson, March 7, 1969

1. Roughly speaking an octonion plane $\mathscr{P}(\mathfrak{O})$ is a plane coordinatized by an octonion (Cayley-Dickson) algebra $\mathfrak{O}$. The most successful approach to these planes has been made by first constructing a reduced exceptional simple Jordan algebra $\mathfrak{Y}=\mathfrak{S}\left(\mathfrak{O}_{3}, \gamma\right)$ and then using $\Im$ to define $P(\mathfrak{O})$. The results on $P(\mathfrak{O})$ in [2], [7], [8], [10] and [13] for $\mathfrak{D}$ an octonion division algebra and those in [9], [11] and [12] for split $\mathfrak{O}$ were obtained along these lines. However, in all of these papers the characteristic of the field is not two. In the present paper, we give a definition of octonion planes based on quadratic Jordan algebras. This definition is valid for all characteristics and both types of octonion algebras. We also indicate how most of the results mentioned above can be derived in this general setting.

We reter the reader to McCrimmon [4] for a definition of a quadratic Jordan algebra. Recall also that the set $\mathfrak{S}\left(\mathfrak{O}_{3}, \gamma\right)$ of 3 by 3 matrices with entries in $\mathfrak{O}$ which are symmetric with respect to the involution $x \mapsto \gamma^{-1} \bar{x}^{l} \gamma$ (where $\gamma=\operatorname{diag}\left\{\gamma_{1}, \gamma_{2}, \gamma_{3}\right\}, 0 \neq \gamma_{i} \in \Phi$, the field) can be endowed with the structure of a quadratic Jordan algebra. (See [5].) We shall use the notation of [4] and [5] with the exception that we write our operators on the right and define $z V_{x, y}=\{z x y\}$ $=x U_{z, y}$. We remark that $U_{x, y}=U_{x+y}-U_{x}-U_{y}$ where in characteristic not two $U_{x}=2 R_{x}^{2}-R_{x^{2}}$ where $y R_{x}=\frac{1}{2}(x y+y x)$. Also $x^{\#}$ in [5] is roughly the adjoint of the matrix $x$ and $x \times y=(x+y)^{*}-x^{*}-y^{*}$.

2. Let $\Im=\mathfrak{S}\left(\mathfrak{O}_{3}, \gamma\right)$ and let $x_{*}$ and $x^{*}$ be two copies of $\{\alpha x \mid 0 \neq \alpha \in \Phi\}$ where $x \in \mathfrak{Y}$ is of rank one; i.e., $x \neq 0$ but $x^{*}=0$. We define the octonion plane $\mathcal{P}(\Im)$ to have points $x_{*}$ and lines $y^{*}$, where $x$ and $y$ are of rank one, and relations (cf. [9])

(1) $x_{*} \mid y^{*}, x_{*}$ incident to $y^{*}, \quad$ if $V_{y, x}=0$,

(2) $x_{*} \simeq y^{*}, x_{*}$ connected to $y^{*}$, if $T(y, x)=0$,

(3) $x_{*} \simeq y_{*}, x_{*}$ connected to $y_{*}$, if $y \times x=0$,

(4) $x^{*} \simeq y^{*}, x^{*}$ connected to $y^{*}$, if $y \times x=0$.

1 These results are contained in the author's doctoral dissertation written under the guidance of Professor N. Jacobson at Yale University. A more detailed paper is forthcoming. The author was a National Science Foundation Graduate Fellow while at Yale. 
The groups $\Gamma, G$, and $S$ of semisimilarities, similarities, and norm preserving transformations respectively of $\Im$ can be defined as usual. If $W \in \Gamma$ has multiplier $\rho$ and associated isomorphism $s$, then $(x W)^{*}$ $=\rho x^{*} \hat{W}$ and $W^{-1} V_{x, y} W=V_{x \hat{W}, y}$ for $x, y \in \Im$ where $\hat{W}=W^{*-1}$ and $T\left(x W^{*}, y\right)^{s}=T(x, y W)$. In particular, $W$ permutes the elements of rank one and the map $[W]: x_{*} \mapsto(x W)_{*}, x^{*} \mapsto(x \hat{W})^{*}$ preserves the relations (1)-(4). Thus, $W \mapsto W^{\prime}$ defines a homomorphism of $\Gamma$ into the collineation group of $\odot(\Im)$. Let $P \Gamma, P G, P S$ be the images of $\Gamma$, $G, S$ respectively. The fundamental theorem of octonion planes says that $P \Gamma$ is the entire collineation group or, more generally, that any collineation of two octonion planes is induced by a semisimilarity of the quadratic Jordan algebras. Also, since $\mathfrak{S}\left(\mathfrak{D}_{3}, \gamma\right)$ and $\mathfrak{S}\left(\mathfrak{D}_{3}, \gamma^{\prime}\right)$ are norm similar, we see that $\odot(\Im)$ depends only on $\mathfrak{D}$, and we write $P(\mathcal{D})=\mathcal{P}(\Im)$.

The points $u_{*}, v_{*}, w_{*}$ from a three-point if $T(u, v \times w) \neq 0$. Four points form a four-point if each subset of three points is a three-point. A lemma (due to Ferrar for (linear) Jordan algebras) is that if $u_{*}, v_{*}$, $w_{*}$ are a three-point then $u, v, w$ are pairwise orthogonal primitive idempotents in some isotope of $\Im$. This can be used to show

LEMma 1. PS is transitive on three-points while $P G$ is transitive on four-points.

By means of Lemma 1, many of the geometric questions about $P(D)$ can be reduced to consideration of special points and lines. One can show $u_{*}, v_{*}$ are incident to exactly one line if and only if $u_{*} \not v_{*}$. Also, one sees that $u_{*} \simeq v^{*}$ if and only if there exists $w_{*} \mid v^{*}$ with $u_{*} \simeq w_{*}$. In this case, if $x_{*} \mid v^{*}$ then either $u_{*} \simeq x_{*}$ or the unique line through $u_{*}$ and $x_{*}$ is connected to $v^{*}$.

\section{An important result on octonion planes is}

TheOREM 1. PS is a simple group.

The proof of Theorem 1 is based on [1, Lemma 4, p. 39]. By Lemma 1 and other transitivity properties of $P S$, one can show that $P S$ is a primitive permutation group of the lines of $P(D)$. If $u \in \mathcal{Y}$ is of rank one and $v \in \Im$ with $T(u, v)=0$, then the map $T_{u, v}=1+V_{u, v}$ $+U_{u} U_{v}$ is a norm preserving map and ${ }^{\lceil} T_{u, v}{ }^{\top}$ fixes $u^{*}$. $T_{u, v}$ is called an algebraic transvection and ${ }^{\top} T_{u, v}{ }^{1}$ is called a transvection. The group $H_{u^{*}}$ generated by ${ }^{\top} T_{u, v}{ }^{7}$ for a fixed $u$ is a normal abelian subgroup of the subgroup of $P S$ fixing $u^{*}$. The other conditions of [1, Lemma 4, p. 39], namely that the $H_{u *}$ 's generate $P S$ and that $P S$ be its own derived group follow from 
THEOREM 2. $S$ is generated by algebraic transvections.

The main step in the proof of Theorem 2 is the identification of a certain subgroup of $S$ with a spin group. Let $e$ be a primitive idempotent in $\Im$ and let $\Im_{0}$ be the Peirce 0 -space of $\Im$ relative to $e$. If $x \in \Im_{0}$, then let $\bar{x} \equiv e \times x \in \Im_{0}$. Also, one has the quadratic form $Q$ on $\Im_{0}$ where $Q(x)=T\left(x^{\sharp}\right)$. We can now state

THEOREM 3. Let $u \in \operatorname{Spin}\left(\Im_{0}, Q\right)$ and write $u=v_{1} v_{2} \cdots v_{2 r}$ with $v_{i} \in \Im_{0}, \Pi Q\left(v_{i}\right)=1$. If $W_{u}=U_{\theta\left(\bar{v}_{1}\right)} U_{\theta\left(v_{2}\right)} \cdots U_{\theta\left(\bar{v}_{2 r-1}\right)} U_{\theta\left(v_{2 r}\right)}$ where $\theta(v)=e+v$, then $u \mapsto W_{u}$ is an isomorphism of $\operatorname{Spin}\left(\Im_{0}, Q\right)$ onto $H$, the group of norm preserving maps fixing $e$ and stabilizing $\Im_{0}$.

A corollary of Theorem 3 is that the group of automorphisms of $\Im$ fixing $e$ is isomorphic to $\operatorname{Spin}\left(\Im_{0}, Q, 1-e\right)$ where in general $\operatorname{Spin}(V$, $Q, c)$ is defined in terms of the Clifford algebra $C(V, Q, c)$ with basepoint much as $\operatorname{Spin}(V, Q)$ is defined in terms of the the Clifford algebra $C(V, Q) . C(V, Q, c)$, which has been studied by Jacobson and $\mathrm{McCrimmon}$ in some unpublished notes, is the tensor algebra on $V$ modulo the ideal generated by $x \otimes x-T(x) x+Q(x) 1$ and $c-1$ where $T(x)=Q(x, c)$ and $Q(c)=1$.

4. $P(\mathfrak{D})$ is a projective plane if and only if $\mathfrak{D}$ is a division algebra. In this case, there are two kinds of involutions in $P G$; those which fix a line of points and those which fix a four-point. In characteristic two, those of the first kind are transvections while those of the second kind are conjugate (under $P S$ ) to an involution induced by an automorphism of $\Im$ which is given by applying an automorphism (of order two) of $D$ to each entry of the 3 by 3 matrix. A useful result is

LEMMA 2. $J$ is an automorphism of order two of $\mathfrak{D}$ in characteristic two if and only if one of the following holds:

(a) $\mathfrak{D}=\mathfrak{B} \oplus \mathfrak{B s}$, where $\mathfrak{B}$ is a totally isotropic subalgebra of $\mathfrak{D}$ relative to the norm $n$ on $\mathfrak{O}, n(1, s)=1$, and $J: b_{1}+b_{2} s \mapsto b_{1}+b_{2} \bar{s}, b_{i} \in \mathfrak{B}$.

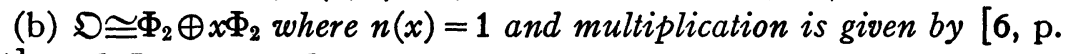
45], and $J$ corresponds to

$$
d_{1}+x d_{2} \mapsto d_{1}+x\left(d_{2} u\right), d_{i} \in \Phi_{2}, u=\left(\begin{array}{ll}
0 & 1 \\
1 & 0
\end{array}\right) .
$$

Lemma 2 is used to show that any isomorphism of groups $P G$ or $P S$ of two projective planes in characteristic two preserves the type of an involution. This, in turn, is used to show that any such isomorphism is induced by a collineation or correlation of the planes. 
5. We call $\pi_{0}: x_{*} \leftrightarrow x^{*}$ the standard polarity on $\odot(\Im)$ where $\Im=\mathfrak{S}\left(\mathfrak{D}_{3}\right)$. We consider the group $P T\left(\pi_{0}\right)$ generated by transvections commuting with $\pi_{0}$. In characteristic two, by using methods similar to those in [13] and [12], one can show that the group $P T\left(\pi_{0}\right)$ is simple and if $\Phi$ has more than two elements then Aut $\Im \cong P T\left(\pi_{0}\right)$. The simplicity of Aut $\Im$ was shown in [3] in the setting of restricted Lie algebras.

\section{REFERENCES}

1. J. Dieudonné, La géométrie des groupes classiques, 2nd ed., Springer-Verlag, Berlin, 1963.

2. N. Jacobson, Some groups of transformations defined by Jordan algebras. III, J. Reine Angew. Math. 207 (1961), 61-85.

3. P. Jonker, Restricted Lie algebras over a field of characteristic 2, Dissertation, Mathematisch Instituut der Rijksuniversiteit te Utrecht, Utrecht, 1968.

4. K. McCrimmon, $A$ general theory of Jordan rings, Proc. Nat. Acad. Sci. U.S.A. 56 (1966), 1072-1079.

5. - The Freudenthal-Springer-Tits constructions of exceptional Jordan algebras, Trans. Amer. Math. Soc. 139 (1969), 495-510.

6. R. D. Schafer, An introduction to non-associative algebras, Academic Press, New York, 1966.

7. T. A. Springer, The projective octave plane, Nederl. Akad. Wetensch. Proc. Ser. A 63 = Indag. Math. 22 (1960), 74-101.

8. T. A. Springer and F. D. Veldkamp, Elliptic and hyperbolic octave planes, Nederl. Akad. Wetensch. Proc. Ser. A 66=Indag. Math. 25 (1963), 413-451.

9. - On Hjelmslev-Moufang planes, Math Z. 107 (1968), 249-263.

10. T. Suh, On isomorphisms of little projective groups of Cayley planes, Nederl. Akad. Wetensch. Proc. Ser. A 65 = Indag. Math. 24 (1962), 320-339.

11. F. D. Veldkamp, Collineation groups in Hjelmslev-Moufang planes, Math. Z. 108 (1968), 37-52.

12. —, Unitary groups in Hjelmslev-Moufang planes, Math. Z. 108 (1969), $288-312$.

13. - Unitary groups in projective octave planes, Compositio Math. 19 (1968), 213-258.

Yale University, New Haven, Connecticut 06520 